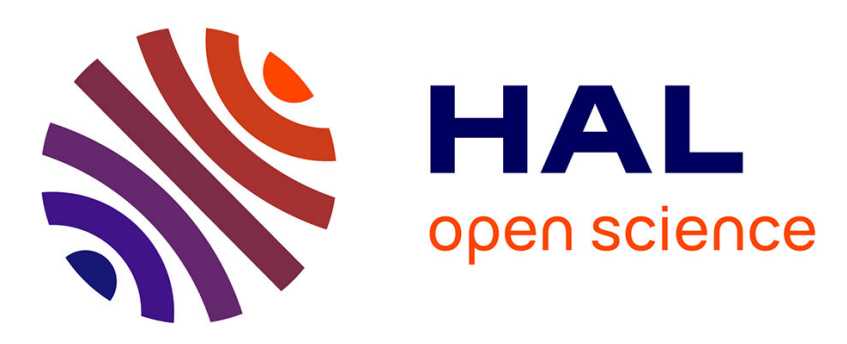

\title{
Self-controlled formation of micro-lenses by optical breakdown inside wide-band-gap materials
}

David Grojo, M. Gertsvolf, H. Jean-Ruel, S. Lei, L. Ramunno, D. M. Rayner, P. B. Corkum

\section{- To cite this version:}

David Grojo, M. Gertsvolf, H. Jean-Ruel, S. Lei, L. Ramunno, et al.. Self-controlled formation of micro-lenses by optical breakdown inside wide-band-gap materials. Applied Physics Letters, 2008, 93, pp.243118. 10.1063/1.3049133 . hal-00341282

\section{HAL Id: hal-00341282 \\ https://hal.science/hal-00341282}

Submitted on 24 Nov 2008

HAL is a multi-disciplinary open access archive for the deposit and dissemination of scientific research documents, whether they are published or not. The documents may come from teaching and research institutions in France or abroad, or from public or private research centers.
L'archive ouverte pluridisciplinaire HAL, est destinée au dépôt et à la diffusion de documents scientifiques de niveau recherche, publiés ou non, émanant des établissements d'enseignement et de recherche français ou étrangers, des laboratoires publics ou privés. 


\title{
Self-controlled formation of micro-lenses by optical breakdown inside wide-band-gap materials
}

\author{
D. Grojo, ${ }^{1}$ M. Gertsvolf, ${ }^{2,1}$ H. Jean-Ruel,${ }^{1}$ S. Lei,${ }^{3}$ L. Ramunno, ${ }^{2}$ D. M. Rayner ${ }^{*},{ }^{1}$ and P. B. Corkum ${ }^{\dagger 2,1}$ \\ ${ }^{1}$ National Research Council, 100 Sussex Drive, Ottawa, ON K1A 0R6, Canada \\ ${ }^{2}$ University of Ottawa, Ottawa, ON K1N 6N5, Canada \\ ${ }^{3}$ Kansas State University, 2012 Durland Hall, Manhattan, KS 66506, USA
}

(Dated: November 24, 2008)

\begin{abstract}
By repeatedly illuminating fused silica slabs with focused femtosecond pulses, we permanently decrease the local refractive index without increasing linear absorption or scattering. This progressively forms a bi-convex lens in the pre-focal region. With linearly polarized light, the index change reaches several percent and is associated with the formation of an array of planar nano-cracks. We analyze the polarization-dependent focusing power of the sub-wavelength periodic structure. While the detailed material modification changes, spontaneous defocusing lens formation is a common feature of every wide band gap transparent materials that we have studied $\left(\mathrm{SiO}_{2}, \mathrm{BK} 7, \mathrm{LiF}\right.$, sapphire, mica).

PACS numbers: 78.67.-n, 81.16.Rf, 33.80.Rv
\end{abstract}

Microfabrication is primarily based on lithographic technologies. These are especially advanced in microelectronics for the fabrication of ultra high-density devices. In the last decade, direct femtosecond laser writing has emerged as an alternative technique for microfabrication inside transparent materials. Focused to small focal spots, intense light pulses make possible to form microscale three-dimensional (3-D) optical elements [1-3].

There are unique advantages to using tightly focused femtosecond pulses. First, multiphoton ionization creates a modest density of free-carriers ( $\cong 10^{20} \mathrm{~cm}^{-3}$ ) that can be controlled with the pulse duration [4]. Second, the nonlinear nature of the laser energy deposition mechanisms confines material change to the focal volume [5]. Third, the pulse power, $P$, is maintained below or near the critical power for self-focusing $\left(P_{C} \cong 1.5 \times 10^{6}\right.$ $\mathrm{W}$ in $\left.\mathrm{SiO}_{2}\right)$. Even in those cases where $P>P_{C}$, beam depletion lowers the power long before the focal region. This is confirmed by the absence of self-phase modulation in the transmitted spectrum [4]. Taken together, the interaction process becomes extraordinary controllable and repeatable.

Experiments show that there are two ways in which the bulk refractive index of transparent materials can be locally modified. At low single pulse fluencies, smooth refractive index increase $\left(\Delta n \approx 10^{-3}\right)$ associated with chemical changes in the material has been extensively studied $[6,7]$. This possibility has been widely exploited to fabricate waveguides and photonic circuits $[1,3,7]$. At higher fluencies the modification generally becomes heterogeneous on a sub-wavelength scale [8-10].

We explore the change in the effective refractive index associated with the modification in the second regime. As we shall see, the modification forms progressively a

\footnotetext{
*David.Rayner@nrc-cnrc.gc.ca

†Paul.Corkum@nrc-cnrc.gc.ca
}

thick microlens with $\approx 30$ micron focal length inside all bulk materials we have investigated: fused and crystalline quartz, LiF, BK7, sapphire, mica. The defocusing of the lens implies a large decrease of the refractive index that can be achieved by nanoscale material disruptions.

We concentrate on fused silica in which the modification takes the form of an array of planar nano-cracks $[11,12]$. These arrays are characterized by their form birefringence [11]. We study the nanoscale material rearrangement that is perfected shot after shot. We demonstrate that as the void inclusions grow throughout the breakdown region, a $2 \%$ refractive index decrease is reached without increasing linear absorption or scattering. This makes the approach suitable for building advanced, high contrast 3D-optical devices in bulk materials.

We use 130 fs laser pulses with peak energy up to few hundred $\mathrm{nJ}$ at wavelength $\lambda_{\text {las }}=800 \mathrm{~nm}$. With our $\mathrm{NA}=0.65$ microscope objective, we would reach a peak intensity of $3 \times 10^{14} \mathrm{~W} \mathrm{~cm}^{-2}$ if the focus were in vac-
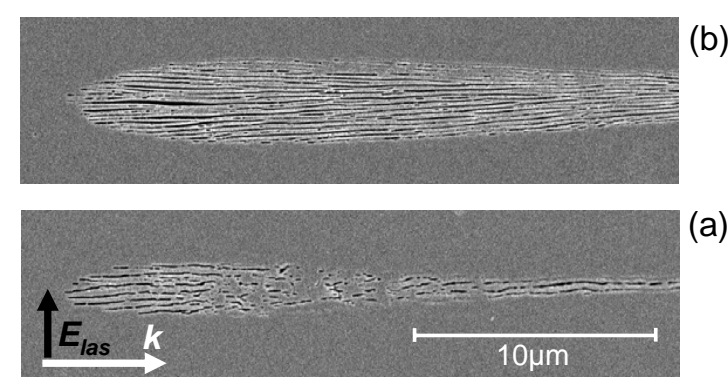

(a)

FIG. 1: Cross-sectional images of the modified regions in fused silica. The modifications were done by repeatedly illuminating bulk materials with $10^{2}$ (a) and $10^{5}$ (b) focused pulses at 350 nJ. Samples are cleaved and etched (1\% HF) to reveal the presence of nano-cracks in bulk by SEM imaging. The relative position of images in the $k$-direction is arbitrary. 


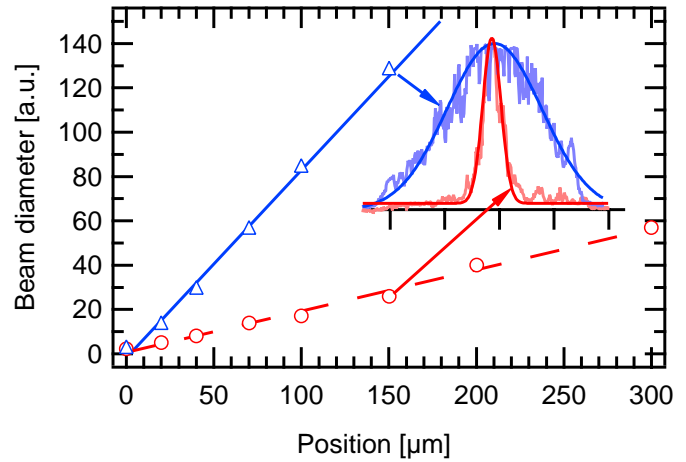

FIG. 2: Measurement of the divergence of the laser beam passing through fresh (solid blue) and modified materials (dashed red) with $10^{7}$ shots laser shots at $350 \mathrm{~nJ}$. The embedded graph shows the normalized beam profiles at a distance of $150 \mu \mathrm{m}$ from the lens.

uum. The threshold intensity for non-linear absorption is $\cong 1.5 \times 10^{13} \mathrm{~W} \mathrm{~cm}^{-2}$ in fused silica [4]. The focus is placed 75 microns under the surface so that spherical aberrations do not affect our focal spot. Figure 1 shows the cross-sectional morphology of modified $\mathrm{SiO}_{2}$ after irradiations with different number of linearly polarized pulses. The modification progressively assumes the shape of a bi-convex micro-lens, determined by the intensity distribution in the focal volume. The size of the lens is controlled by the laser exposure. The scanning electron microscope (SEM) image reveals that the local change in the material optical properties is caused by the formation of a self-organized array of nanoplanes that have been identified as void nano-cracks $\cong 10 \mathrm{~nm}$ wide) [13]. These stretch through the breakdown region in planes that have their normal along the laser field direction. The sub-wavelength periodic structure $(\cong 250$ $\mathrm{nm}$ spacing) is then accompanied by birefringence and a lower refractive index.

We estimate the average refractive index modification by measuring the change in divergence of a low intensity beam that passes through the modified region. The measurement of the beam divergence is shown in figure 2. Experimentally we use a second, higher numerical aperture $(\mathrm{NA}=0.75)$ microscope objective to collect and collimate the light transmitted through the sample. We focus $\left(f_{\text {lens }}=50 \mathrm{~mm}\right)$ the collimated beam on a CCD. As we translate the imaging system along the optical axis, we measure the changes in the size (FWHM) of the Gaussian beam on the CCD. The top curve in figure 2 corresponds to the response of the fresh material. The bottom curve was obtained when the material was previously exposed to $10^{7}$ laser shots at $350 \mathrm{~nJ}$. The divergence decreases by a factor of five for the beam propagating through a modified region. According to the bi-convex shape of the modification, the large defocusing is associated with a large decrease of the refractive index.

Form birefringence arises because of the anisotropic ordered arrangement of transparent materials on a scale

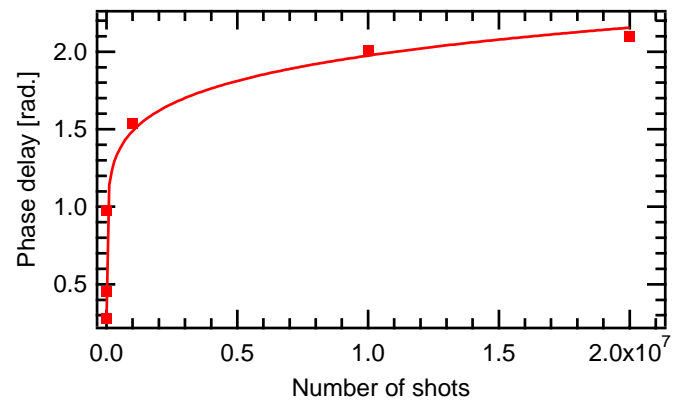

FIG. 3: Phase delay $\Delta \phi=2 \pi\left(n_{s}-n_{p}\right) d / \lambda_{\text {las }}$ measured between $p-$ and $s$ - polarized light propagating trough the modified material. The measurements are performed after modification of $\mathrm{SiO}_{2}$ with different number of laser shots $(E=350$ $\mathrm{nJ}$ ). The growth of birefringence correlates with the subwavelength periodic arrangement that is perfected (Fig.1) on a shot-to-shot basis.

smaller than the wavelength. Considering the idealized case of infinite periodic planar void cracks (thickness $t_{1}$ ) in fused silica (thickness $t_{2}$ ), the treatment of the form birefringence problem shows that the structure behaves like a uniaxial crystal where $n_{p}$ (resp. $n_{s}$ ) is the effective index for an incident field perpendicular (resp. parallel) to the planes [14]. If $n=1.45, t_{1}=8 \mathrm{~nm}, t_{2}=200 \mathrm{~nm}$ that is $R=t_{1} / t_{2}=4 \%$, all typical numbers from figure 1 , we obtain $n_{p}=n \sqrt{(R+1) /\left(R n^{2}+1\right)} \cong 1.42$ and $n_{s}=$ $n \sqrt{\left(R / n^{2}+1\right) /(R+1)} \cong 1.435$. The formation of the cracks with sub-wavelength periodicity should therefore be accompanied by a polarization dependent-decrease of the refractive index exceeding $2 \%$.

We measure form birefringence in fused $\mathrm{SiO}_{2}$ using a low intensity probe linearly polarized at 45 relative to the writing laser polarization (p). By analyzing the polarization state of the transmitted beam, we obtain, the phase delay between $p-$ and $s-$ polarized light, $\Delta \phi$, as a function of the number of shots (Fig. 3). We find that the polarization changes gradually from linear to circular as the number of shots of the writing laser increases. Accordingly, over the $d \cong 25 \mu \mathrm{m}$ long interaction region, the phase delay exceeds that of a quarter-wave plate for a $10^{7}$ shot exposure. It corresponds to a polarization sensitivity of the refractive index $\Delta n=n_{p}-n_{s}=\Delta \phi \lambda_{\text {las }} / 2 \pi d \cong-1 \%$ which is in reasonable agreement with our form birefringence predictions (see above).

Based on the birefringence properties, one would expect also a polarization dependence in the power of the lens. However, the phase advance implied by this selfgenerated lens is much greater than the relative phase advance of $s-$ and $p-$ polarized light. Accordingly, we observe very little difference between polarizations when we characterize the large defocusing power of the lens (as in Fig.2).

We can probe the self-formed lens spatially by performing a $z$-scan of the refraction properties of the modified 


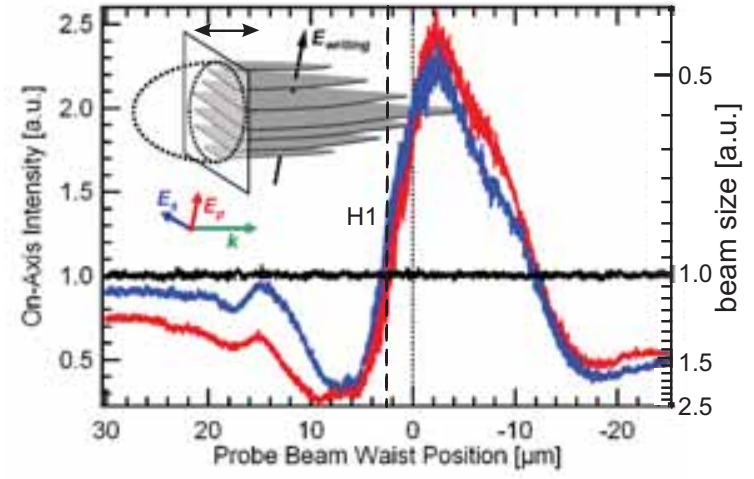

FIG. 4: (color online) $z$-scan of the refraction properties of the modified structure using $p-$ (red) and $s-$ (blue) polarized probes. The embedded image shows the geometry of modifications that is probed. The spot size, or divergence, of the probe beam (right ordinate) is directly evaluated by measuring the probe energy transmitted by an on-axis aperture (left ordinate). Signal level 1 corresponds to $40 \%$ transmission by the aperture of the laser beam passing through a fresh material. The measurement shows a polarization dependent refraction at the first interface of the structure.

region for $p-$ (writing) and $s-$ polarized light. We focus the low intensity probe light with the microscope objective inside the modified region and measure the energy in the central region of the transmitted beam, using an on-axis aperture. The size of the aperture is set to transmit $40 \%$ of the laser beam, when it propagates through a fresh material. This provides for the optimum sensitivity of the z-scan. Translating the focusing objective along the optical axis changes the beam divergence and, therefore, the signal amplitude on the photodiode. Using this approach we can separate between the influences of the back and the front surfaces of the lens.

Figure 4 shows the measured signal as a function of the probe position for a lens written with $10^{7}$ shots at 350 nJ. The zero position corresponds to the probe beam focusing exactly at the same location as the writing beam. The intensity measured in this situation is much larger than that measured for an unmodified material (black - unity level). This is clear evidence of a decrease in divergence. By moving the probe focus progressively toward the front-curvature of the lens (positive values), the signal becomes lower than unity when the beam waist crosses the first principal plane $\mathrm{H} 1$ of the formed lens. The full scan measurement shows that form birefringence is present at the input interface of the modified region. As we move closer to the front of the lens, the measured signal becomes highly polarization dependent. As predicted (see above), a stronger refraction occurs for the writing polarization, $p$. The polarization sensitivity vanishes with the sharp exit tip where the number of nano-planes drops to only a few (scan toward negative $z$-positions).
The creation of the defocusing microlens in the interaction region in fused silica does not necessarily require the formation of a birefringent structure. Similar divergence change is found when circularly polarized light is used for lens writing. In this case, the nano-cracks are quasi-random in shapes and location [8]. In general, the creation of multiple sub-wavelength size voids inside the bulk leads to the reduction of the volume refractive in$\operatorname{dex}, \Delta n \approx R(1-n)$ where $R$ is the volume fill ratio. We repeated the lens writing experiment in other transparent dielectrics (crystalline $\mathrm{SiO}_{2}, \mathrm{BK} 7, \mathrm{LiF}$, sapphire and mica). We found similar increase in the signal through an on-axis aperture, (by a factor of 1.5 to 2) in all of them. Although the formation of the birefringent structures has not yet been reported for other dielectrics, this large divergence decrease implies that nano-cracking and subsequent lens formation is likely to be a general phenomenon for dielectrics.

While we have concentrated on self-formed microlenses and their potential technological benefits, the underlying material science is only partially understood. We will focus a future paper on this aspect. One tool to understanding and monitoring material modification in real time is to look at the non-linear absorption of each individual pulse that creates the structure. Shot after shot, an increase of the transmission associated with reduced multiphoton absorption is systematically observed for both linearly and circularly polarized light. Like lens formation, enhanced transmission is found in all wide band gap materials that we have studied under repeated illumination. In fused silica, it is also a polarization dependent process. The feedback effect of the defocusing lens on the beam that creates it and the local field distribution in the progressively structured materials are contributions to this self-limitation of the modification. The saturation of the birefringence properties with the number of laser shots shown in figure 3 is another manifestation of the self-limited character of the lens fabrication process. It is an attractive feature for technological considerations.

Finally, our results emphasize that efficient polarization-dependent component such as wave-plates, retarders and couplers can be efficiently fabricated provided that the ordered and self-limited material rearrangement occurs on a scale much smaller than the component size. These capabilities open a route to integrating advanced functionality inside optical fibers or bulk materials.

\section{Acknowledgments}

The authors are very grateful to Eli Simova, Wayne Boland and Jeffrey Fraser for sample preparation and SEM imaging. 
[1] K. M. Davis, K. Miura, N. Sugimoto, and K. Hirao, Opt. Lett. 21, 1729 (1996).

[2] R. R. Gattass and E. Mazur, Nature Photonics 2, 219 (2008).

[3] K. Miura, J. R. Qiu, H. Inouye, T. Mitsuyu, and K. Hirao, Appl. Phys. Lett. 71, 3329 (1997).

[4] D. M. Rayner, A. Naumov, and P. B. Corkum, Opt. Exp. 13, 3208 (2005).

[5] A. P. Joglekar, H. H. Liu, E. Meyhofer, G. Mourou, and A. J. Hunt, PNAS 101, 5856 (2004).

[6] J. W. Chan, T. Huser, S. Risbud, and D. M. Krol, Opt. Lett. 26, 1726 (2001).

[7] D. Homoelle, S. Wielandy, A. L. Gaeta, N. F. Borrelli, and C. Smith, Opt. Lett. 24, 1311 (1999).

[8] R. S. Taylor, E. Simova, and C. Hnatovsky, Opt. Lett. 33, 1312 (2008).
[9] P. G. Kazansky, H. H. Inouye, T. Mitsuyu, K. Miura, J. Qiu, and F. Hirao, K.and Starrost, Phys. Rev. Lett. 82, 2199 (1999).

[10] Y. Shimotsuma, P. G. Kazansky, J. Qiu, and K. Hirao, Phys. Rev. Lett. 91, 247405 (2003).

[11] E. Bricchi, B. G. Klappauf, and P. G. Kazansky, Opt. Lett. 29, 119 (2004).

[12] V. R. Bhardwaj, E. Simova, P. P. Rajeev, C. Hnatovsky, R. S. Taylor, D. M. Rayner, and P. B. Corkum, Phys. Rev. Lett. 96 (2006).

[13] C. Hnatovsky, R. S. Taylor, P. P. Rajeev, E. Simova, V. R. Bhardwaj, D. M. Rayner, and P. B. Corkum, Appl. Phys. Lett. 87, 014104 (2005).

[14] M. Born and E. Wolf, Principles of Optics (Cambridge, University Press, 1999). 